\title{
Double-edged Sword Role of Iron-loaded Ferritin in Extracellular Vesicles
}

\author{
Shinya Toyokuni ${ }^{1}{ }^{2}$, Yingyi Kong', Hao Zheng', Danyang Mi', Misako Katabuchi', Yashiro Motooka', Fumiya Ito ${ }^{1}$ \\ ${ }^{1}$ Department of Pathology and Biological Responses, Nagoya University Graduate School of Medicine, ${ }^{2}$ Center for Low- \\ temperature Plasma Sciences, Nagoya University, Nagoya, Japan
}

\begin{abstract}
Human epidemiological and animal studies have demonstrated that excess iron is a risk for cancer. The responsible mechanisms are: 1) increased intracellular iron catalyzes the Fenton reaction to generate hydroxyl radicals, leading to mutagenic oxidative DNA lesions; 2) iron is necessary for cellular proliferation as cofactors of many enzymes. Thus, iron-excess milieu promotes selecting cellular evolution to ferroptosis-resistance, a major basis for carcinogenesis. Ferritin is a 24-subunit nanocage protein required for iron storage under the regulation of the iron-regulatory protein (IRP)/iron-responsive element (IRE) system. Ferritin is a serum marker, representing total body iron storage. However, how ferritin is secreted extracellularly has been unelucidated. We recently discovered that an exosomal marker CD63 is regulated by the IRP/IRE system and that iron-loaded ferritin is secreted as extracellular vesicles under the guidance of nuclear receptor coactivator 4 (NCOA4). On the other hand, we found that macrophages under asbestos-induced ferroptosis emit ferroptosis-dependent extracellular vesicles (FedEVs), which are received by nearby mesothelial cells, resulting in significant mutagenic DNA damage. Therefore, cells, including macrophages, can share excess iron with other cells, via iron-loaded ferritin packaged in extracellular vesicles as safe non-catalytic iron. However, similar process, such as one involving FedEVs, may cause accumulation of excess iron in other specific cells, which may eventually promote carcinogenesis.
\end{abstract}

Key Words Ferritin, Extracellular vesicles, Iron, Ferroptosis, Asbestos

\section{INTRODUCTION}

The first life on earth was born under the ancient sea of abundant $\mathrm{Fe}(\mathrm{II}) 3.8$ Gya $[1,2]$, and it is generally accepted that no independent life on earth can live without iron [3]. Iron is a major redox-active transition metal, which is used for various electron transfer reactions in the form of $\mathrm{Fe}(\mathrm{II})$, Fe-S cluster or heme [4]. During evolution, the life obtained the sulfhydryl systems [5], including glutathione, to counteract iron toxicity when present in excess, which might have been just insoluble FeS at first $[6,7]$. Finally, the life acquired the capacity to use molecular oxygen, which allows more flexible and versatile transfer of one to four electron(s) at a time and continuous electron flow inside the entire cells (Fig. 1) [8].

\section{IRON AND OXYGEN FOR ELECTRON TRANSFER}

During the $\mathrm{O}_{2}$ metabolism, reactive oxygen species are in- evitably produced. Superoxdie $\left(\mathrm{O}_{2}^{-}\right)$and hydrogen peroxide $\left(\mathrm{H}_{2} \mathrm{O}_{2}\right)$ are generated via enzymatic reactions whereas hydroxyl radical is produced via the chemical reaction called the Fenton reaction $\left(\mathrm{Fe}[\mathrm{II}]+\mathrm{H}_{2} \mathrm{O}_{2} \rightarrow \mathrm{Fe}[\mathrm{III}]+\cdot \mathrm{OH}+\mathrm{OH}^{-}\right.$) (Fig. 1) [9-11]. Catalytic $\mathrm{Fe}(\mathrm{II})$ is indeed dangerous for life when present unconfined. Now we can detect catalytic Fe(II) by use of turn-off or turn-on fluorescent probes in cellular experiments [12-14]. Fortunately, we have several distinct mechanisms to minimize this iron toxicity.

We fully utilize the advantageous characteristics of iron. $\mathrm{Fe}(\mathrm{III})$ is practically insoluble at neutral $\mathrm{pH}$ whereas $\mathrm{Fe}(\mathrm{II})$ is soluble [11]. Iron is transported by transferrin in higher organisms as $\mathrm{Fe}$ (III) [15]. Fe(III) has to be reduced to $\mathrm{Fe}(\mathrm{II})$ to be transported through the biomembranes with various transporters, such as divalent metal transporter 1 (DMT1) (solute carrier family 11 member 2 [SLC11A2]) [16] and ferroportin (solute carrier family 40 member 1 [SLC40A1]) [17]. Iron absorption through the duodenal mucosa is strictly regulated via iron storage status [18]. Excess iron in the cell is stored in

Received November 19, 2021, Revised December 8, 2021, Accepted December 14, 2021

Correspondence to Shinya Toyokuni, E-mail: toyokuni@med.nagoya-u.ac.jp, https://orcid.org/0000-0002-5757-1109

Check for updates 


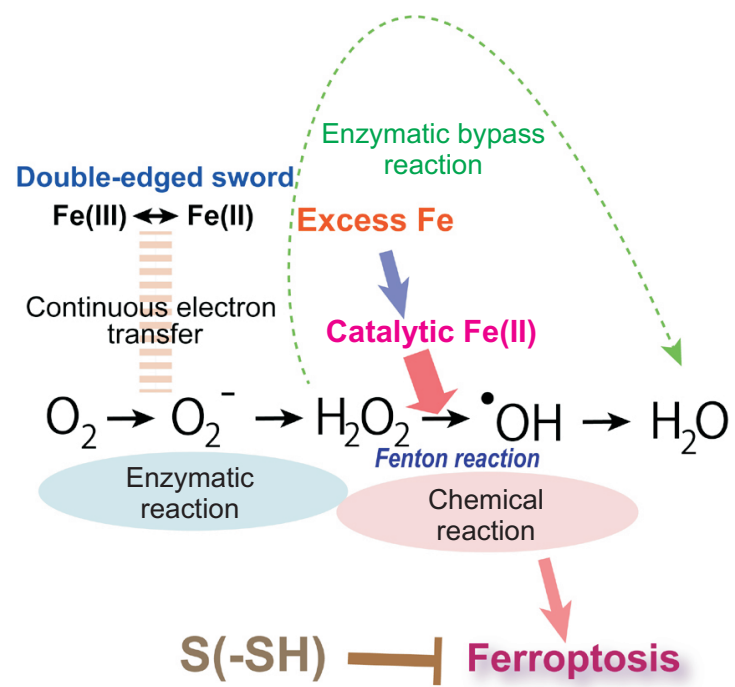

Figure 1. Molecular oxygen as versatile electron acceptors, and its association with ferroptosis. Superoxide and hydrogen peroxide are generated through various enzymatic reactions whereas hydroxyl radicals are produced via chemical reactions. Enzymatic bypass reactions indicate direct decomposition of hydrogen peroxide to water by a variety of enzymes, such as catalase and glutathione peroxidase. Hydroxyl radicals are closely associated with ferroptosis, which is counteracted by sulfhydryls (-SH). cytosol as ferritin in the form of $\mathrm{Fe}(\mathrm{III})$. Furthermore, recently identified iron chaperones play roles in the safe transport of $\mathrm{Fe}(\mathrm{II})$ in the cytosol. These chaperones are poly $\mathrm{rC}$ binding protein 1 (PCBP1) and PCBP2, which were first reported as nuclear proteins responsible for transcriptional regulation $[19,20]$. Both PCBPs accept 3 molecules of $\mathrm{Fe}(\mathrm{II})$ and the resulting complexes are redox-inactive. Whereas PCBP2 can accept Fe(II) from DMT1 [21] and heme oxygenase 1 [22] and can transfer $\mathrm{Fe}$ (II) to ferroportin [23], PCBP1 can load $\mathrm{Fe}$ (II) to ferritin (Fig. 2) [24]. PCBP1 and PCBP2 antagonize to each other, where PCBP1 works as a tumor suppressor $[25,26]$ and PCBP2 as an oncogene in general $[27,28]$. Ferritinophagy, a form of autophagy, is used to retrieve $\mathrm{Fe}(\mathrm{III})$ from ferritin cores, which is reduced in lysosome to $\mathrm{Fe}$ (II) via six-transmembrane epithelial antigen of prostate 3 (STEAP3) [29].

\section{IRON-DEPENDENT EXCRETION OF FERRITIN AS EXTRACELLULAR VESICLES}

Ferritin is a 24-subunit nanocage protein, consisting of light chain (FTL) and heavy chain (FHL), for safe iron storage under the regulation of the iron-regulatory protein (IRP)/ iron-responsive element (IRE) posttranscriptional system. Ferritin is also a serum marker representing body iron storage [30]. However, how ferritin is secreted extracellularly has been completely unknown in the absence of secretory signal peptides [31]. We recently discovered for the first time that an

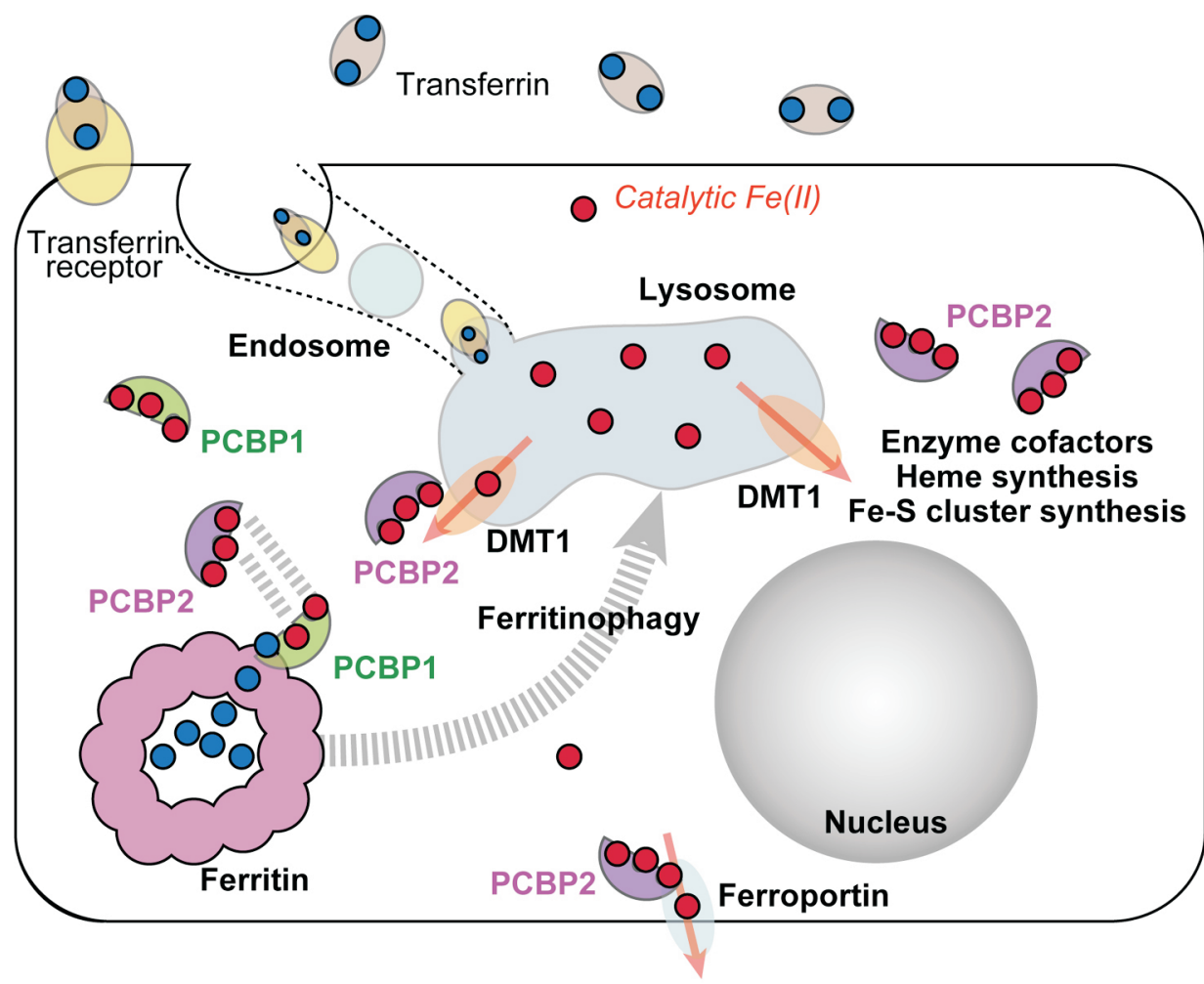

Figure 2. Cytosolic iron chaperon systems. Iron is incorporated by binding of transferrin to transferrin receptor into the cellular cytosol through late endosome/lysosome. Iron retrieved and reduced by STEAP3 (sixtransmembrane epithelial antigen of the prostate 3) to $\mathrm{Fe}(\mathrm{II})$ in late endosome/ lysosome goes through divalent metal transporter 1 (DMT1) (solute carrier family 11 member 2 [SLC11A2]) to cytosol, where $\mathrm{Fe}(\mathrm{II})$ is immediately chaperoned by poly $r C$ binding protein 2 (PCBP2). PCBP1 is another Fe(II) chaperone, competing each other. PCBP1 can load iron to ferritin, where $\mathrm{Fe}(\mathrm{II})$ is oxidized to $\mathrm{Fe}(\mathrm{III})$ via ferritin heavy chain (FTH). PCBP2 appears to play wider roles in transporting $\mathrm{Fe}(\mathrm{II})$ in the cytosol. If $\mathrm{Fe}(\mathrm{II})$ is not chaperoned by PCBP1/2 due to pathologic causes, unchaperoned $\mathrm{Fe}(\mathrm{II})$ is catalytic. Red filled circle, Fe(II); blue filled circle, $\mathrm{Fe}(\mathrm{III})$. Note that $\mathrm{Fe}(\mathrm{III})$ is insoluble at neutral $\mathrm{pH}$. 
exosomal marker CD63 is regulated by the IRP/IRE system and that iron-loaded ferritin is secreted as extracellular vesicles (EVs) with the guidance of nuclear receptor coactivator 4 (NCOA4) [32]. This is an efficient sustainable mechanism to share excess iron with nearby or distant cells in a safe manner (Fig. 3).

\section{EXCESS IRON AND CARCINOGENESIS}

It is well known that iron metabolism is a semi closed system for an individual in higher animals. Namely, the daily fraction of incomings and outgoings is extremely small in comparison to the total amount $(\sim 0.025 \%)$. In humans, adult males retain $\sim 4 \mathrm{~g}$ of iron in total whereas premenopausal females have $\sim 2.5 \mathrm{~g}$ of iron [33]. There is no active pathway to excrete iron to the outside of the body. The only exceptions are hemorrhage or phlebotomy to lose red blood cells, and the use of redox-inactive iron chelating agents, such as desferal, deferasirox and deferiprone [34]. Accordingly, iron would be present in excess during aging by the decrease in metabolic rate, causing ferroptosis time-dependently in a fraction of cells in various organs of rats [35].

Excess iron has also been associated with carcinogenesis, according to the human epidemiological study of the general population or specific diseases, including genetic hemochromatosis and ovarian endometriosis [36-38] as well as various animal studies $[4,8,39]$. The responsible mechanisms are: 1) increased intracellular iron catalyzes Fenton reaction to gen-

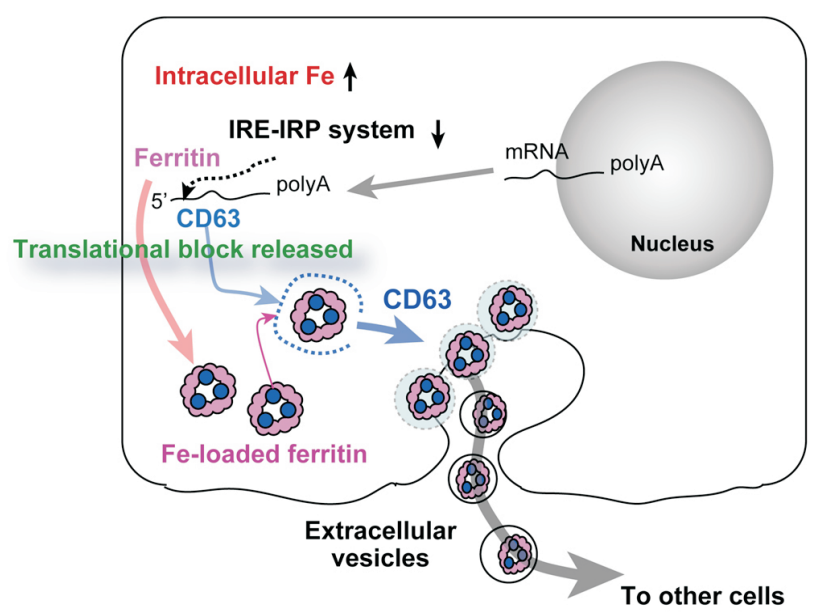

Figure 3. Iron-loaded ferritin is secreted through extracellular vesicles under the regulation of IRP/IRE in CD63. CD63 is one of the exosomal markers. In iron excess conditions, not only an iron storage protein, ferritin, but also CD63 is induced. CD63 recruits nuclear receptor coactivator 4 (NCOA4) to pack iron-loaded ferritin to release as extracellular vesicles. Of note, $C D 63$ gene was discovered to possess the IRE consensus sequence at the 5' untranslated region of mRNA, similar to that of ferritin gene, which blocks the translation of CD63 in the presence of ample amount of iron under the IRE/IRP system. IRE, iron-responsive element; IRP, iron-regulatory protein. Refer to text and the reference [32] for details. erate hydroxyl radicals, leading to mutagenic oxidative DNA lesions [40-42]; 2) iron is necessary for cellular proliferation as cofactors of many enzymes $[4,8,10,33,43,44]$.

\section{EXCRETION OF FERRITIN AS BY-PRODUCT OF ASBESTOS-INDUCED FERROPTOSIS}

Asbestos, a natural nanofibrous mineral, is still used worldwide, especially in some Asian countries, Russia, and South America due to the economic merits, in spite of the designation by World Health Organization as a definite human carcinogen (Group 1 by International Agency for Research on Cancer [IARC]) [45]. Asbestos is resistant to heat, acid and friction, and is also flexible for various industrial use. The association of asbestos exposure and mesothelioma is well established $[45,46]$. Asbestos is inhaled through the airway to the pulmonary parenchyma. However, the major target cells for carcinogenesis are parietal mesothelial cells in the pleural cavity, which has been a long mystery to be solved $[47,48]$.

Molecular mechanisms underlying asbestos-induced mesothelioma have been intensively studies for these two decades. The important point is that the biopersistent nanofibers go through pulmonary parenchyma, by penetrating visceral pleura, into the pleural cavity after collecting hemoglobin originating from red blood cells on the surface and hence iron, depending on the negative pressure of the cavity, and then injures the parietal pleural mesothelial cells $[48,49]$. Amazingly, this process requires a few decades.

The ability of mesothelial cells to phagocytose asbestos fibers provides a high risk for DNA double strand breaks and the resultant mutations because asbestos fibers present a high affinity for histones as well $[50,51]$. The tumor suppressor $p 16^{\text {INK4a }}$ is activated in response to DNA damage as well as oxidative stress. Therefore, it is no wonder that homozygous deletion of $p 16^{\text {INK4a }}$ is the major mutation observed in human and rat mesothelioma [52,53], representing direct DNA double-strand breaks by asbestos fiber in mesothelial cells and the following erroneous end-joining of DNA strands $[47,48]$. Of note, homozygous deletion of $p 16^{I N K 4 a}$ is the major target mutation also in the Fenton reaction-induced renal cell carcinoma in rats [42]. However, the role of macrophages in mesothelial carcinogenesis has not been clear other than what is called "frustrated phagocytosis [54]."

According to our 2020 report, macrophages generate mutagenic milieu for the surface mesothelial cells via ferroptosis, catalytic Fe(II)-dependent regulated necrosis accompanied by lipid peroxidation, upon taking up asbestos fibers as foreign material [55]. We have further sublimated this concept into a more concrete one in 2021 that macrophages under asbestos-induced ferroptosis emit ferroptosis-dependent extracellular vesicles (FedEVs) [56] which are received by mesothelial cells, resulting in significant mutagenic DNA lesions (Fig. 4).

Therefore, various cells, including macrophages, can share 


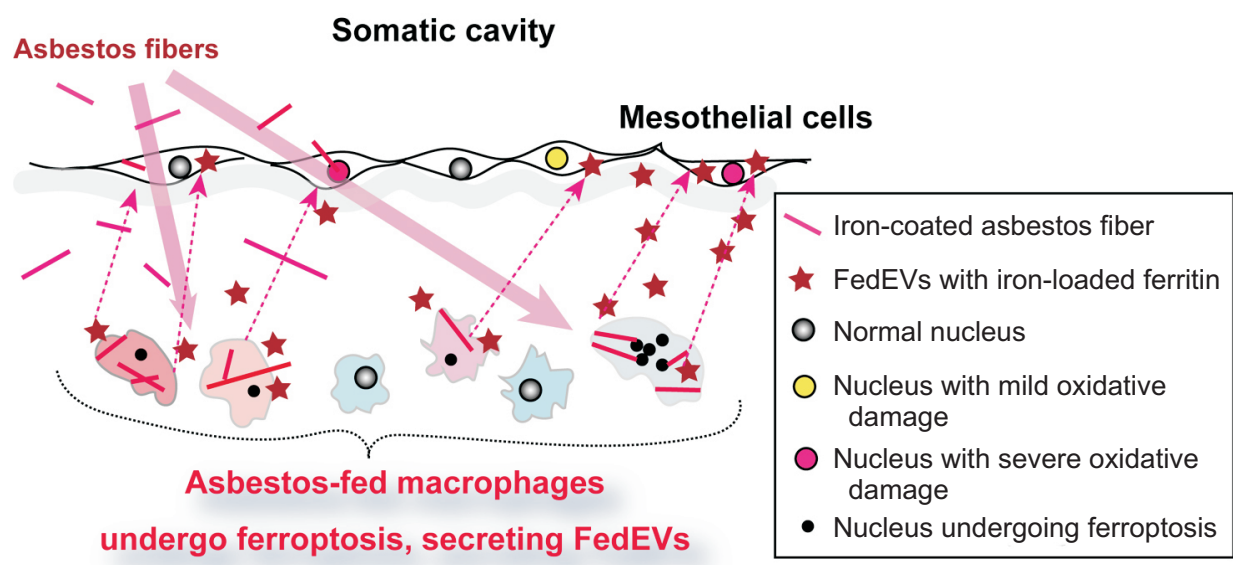

Figure 4. Asbestos-induced ferroptosis in macrophages emits ferroptosis-dependent extracellular vesicles (FedEVs; filled stars), which is received by surface mesothelial cells resulting in oxidative DNA damage. Note that there are direct and indirect damage by asbestos fibers to surface mesothelial cells in the somatic cavities. Macrophages with pyknotic nucleus are undergoing ferroptosis while the other macrophages are alive. Mesothelial cells with yellow/red filled nucleus present mild/severe oxidative damage in the genomic DNA, predisposing to carcinogenesis.

excess iron with the other cells of different types via ferritin in extracellular vesicles as safe non-catalytic Fe(III). However, a similar process, one involving FedEVs, may cause accumulation of excess iron in other specific cells, which may eventually contribute to carcinogenesis.

\section{CONCLUSION}

The major causes of human mortality in most countries are cancer and atherosclerosis (myocardial infarction and cerebral infarction/hemorrhage) except for emerging infectious diseases, such as COVID-19. We believe that these conditions, especially cancer, are associated with the long-term use of iron and oxygen [4]. Thus, modifying iron metabolism would be important as a practical way to prevent carcinogenesis. The present finding on the role of EVs in the transport of iron-loaded ferritin is important to consider future strategy for cancer prevention. We have been using plastics thus far so much in our daily life for convenience, which currently causes microplastics and nanoplastics pollution in the sea [57]. Surprisingly, the microplastics and nanoplastics are coming back to us as part of seafood diet [58], which is an emergent issue in current ecotoxicology to be further explored from the viewpoint of iron and foreign body.

\section{ACKNOWLEDGMENTS}

This work was supported, in part, by JST CREST (Grant No. JPMJCR19H4), JSPS Kakenhi (Grant No. JP19H05462 and JP2OH05502) and Research Grant of the Princess Takamatsu Cancer Research Fund (19-251) to ST.

\section{CONFLICTS OF INTEREST}

No potential conflicts of interest were disclosed.

\section{ORCID}

Shinya Toyokuni, https://orcid.org/0000-0002-5757-1109

Yingyi Kong, https://orcid.org/0000-0002-6481-0745

Hao Zheng, https://orcid.org/0000-0002-2821-4153

Danyang Mi, https://orcid.org/0000-0003-2035-0596

Misako Katabuchi, https://orcid.org/0000-0003-2998-6056

Yashiro Motooka, https://orcid.org/0000-0002-4230-7559

Fumiya Ito, https://orcid.org/0000-0001-5310-9630

\section{REFERENCES}

1. Olson KR, Straub KD. The role of hydrogen sulfide in evolution and the evolution of hydrogen sulfide in metabolism and signaling. Physiology (Bethesda) 2016;31:60-72.

2. Gualtieri AF, Andreozzi GB, Tomatis M, Turci F. Iron from a geochemical viewpoint. Understanding toxicity/pathogenicity mechanisms in iron-bearing minerals with a special attention to mineral fibers. Free Radic Biol Med 2019;133:21-37.

3. Knoll AH, Nowak MA. The timetable of evolution. Sci Adv 2017;3:e1603076.

4. Toyokuni S, Kong Y, Cheng Z, Sato K, Hayashi S, Ito F, et al. Carcinogenesis as side effects of iron and oxygen utilization: from the unveiled truth toward ultimate bioengineering. Cancers (Basel) 2020;12:3320.

5. Sies $\mathrm{H}$. Glutathione and its role in cellular functions. Free Radic Biol Med 1999;27:916-21.

6. Pósfai M, Buseck PR, Bazylinski DA, Frankel RB. Reaction sequence of iron sulfide minerals in bacteria and their use as biomarkers. Science 1998;280:880-3.

7. Schippers A, Jorgensen BB. Biogeochemistry of pyrite and iron sulfide oxidation in marine sediments. Geochim Cosmochim Acta 2002;66:85-92

8. Toyokuni S, Yanatori I, Kong Y, Zheng H, Motooka Y, Jiang L. Ferroptosis at the crossroads of infection, aging and cancer. Cancer Sci 2020;111:2665-71.

9. Fenton $\mathrm{HJH}$. Oxidation of tartaric acid in presence of iron. $\mathrm{J}$ 
Toyokuni et al.

Chem Soc Trans 1894;65:899-910.

10. Toyokuni S. Iron-induced carcinogenesis: the role of redox regulation. Free Radic Biol Med 1996;20:553-66.

11. Koppenol WH, Hider RH. Iron and redox cycling. Do's and don'ts. Free Radic Biol Med 2019;133:3-10.

12. Hirayama T, Okuda K, Nagasawa H. A highly selective turn-on fluorescent probe for iron(II) to visualize labile iron in living cells. Chem Sci 2013;4:1250-6.

13. Mukaide T, Hattori Y, Misawa N, Funahashi S, Jiang L, Hirayama $\mathrm{T}$, et al. Histological detection of catalytic ferrous iron with the selective turn-on fluorescent probe RhoNox-1 in a Fenton reaction-based rat renal carcinogenesis model. Free Radic Res 2014;48:990-5

14. Hirayama T. Fluorescent probes for the detection of catalytic Fe(II) ion. Free Radic Biol Med 2019;133:38-45.

15. Kawabata $H$. Transferrin and transferrin receptors update. Free Radic Biol Med 2019;133:46-54.

16. Yanatori I, Kishi F. DMT1 and iron transport. Free Radic Biol Med 2019;133:55-63.

17. Ganz T. Erythropoietic regulators of iron metabolism. Free Radic Biol Med 2019;133:69-74.

18. Gunshin H, Mackenzie B, Berger UV, Gunshin Y, Romero MF, Boron WF, et al. Cloning and characterization of a mammalian proton-coupled metal-ion transporter. Nature 1997;388:482-8.

19. Philpott CC, Jadhav S. The ins and outs of iron: escorting iron through the mammalian cytosol. Free Radic Biol Med 2019;133:112-7.

20. Yanatori I, Richardson DR, Toyokuni S, Kishi F. The new role of poly $(\mathrm{rC})$-binding proteins as iron transport chaperones: proteins that could couple with inter-organelle interactions to safely traffic iron. Biochim Biophys Acta Gen Subj 2020;1864:129685.

21. Yanatori I, Yasui Y, Tabuchi M, Kishi F. Chaperone protein involved in transmembrane transport of iron. Biochem $J$ 2014;462:25-37.

22. Yanatori I, Richardson DR, Toyokuni S, Kishi F. The iron chaperone poly( $\mathrm{rC}$ )-binding protein 2 forms a metabolon with the heme oxygenase 1/cytochrome P450 reductase complex for heme catabolism and iron transfer. J Biol Chem 2017;292:1320529.

23. Yanatori I, Richardson DR, Imada K, Kishi F. Iron export through the transporter ferroportin 1 is modulated by the iron chaperone PCBP2. J Biol Chem 2016;291:17303-18.

24. Shi H, Bencze KZ, Stemmler TL, Philpott CC. A cytosolic iron chaperone that delivers iron to ferritin. Science 2008;320:120710.

25. Guo J, Jia R. Splicing factor poly(rC)-binding protein 1 is a novel and distinctive tumor suppressor. J Cell Physiol 2018;234:33-41.

26. Zhang Y, Meng L, Xiao L, Liu R, Li Z, Wang YL. The RNAbinding protein PCBP1 functions as a tumor suppressor in prostate cancer by inhibiting mitogen activated protein kinase 1 . Cell Physiol Biochem 2018;48:1747-54.

27. Chen C, Lei J, Zheng Q, Tan S, Ding K, Yu C. Poly(rC) binding protein 2 (PCBP2) promotes the viability of human gastric cancer cells by regulating CDK2. FEBS Open Bio 2018;8:764-73.
28. Wang X, Guo Q, Wang H, Yuan X, Wang B, Lobie PE, et al. $P C B P 2$ posttranscriptional modifications induce breast cancer progression via upregulation of UFD1 and NT5E. Mol Cancer Res 2021;19:86-98.

29. Mancias JD, Wang X, Gygi SP, Harper JW, Kimmelman AC. Quantitative proteomics identifies NCOA4 as the cargo receptor mediating ferritinophagy. Nature 2014;509:105-9.

30. Wish JB. Assessing iron status: beyond serum ferritin and transferrin saturation. Clin J Am Soc Nephrol 2006;1 Suppl 1:S48.

31. Truman-Rosentsvit M, Berenbaum D, Spektor L, Cohen LA, Belizowsky-Moshe S, Lifshitz $L$, et al. Ferritin is secreted via 2 distinct nonclassical vesicular pathways. Blood 2018;131:34252.

32. Yanatori I, Richardson DR, Dhekne HS, Toyokuni S, Kishi F. CD63 is regulated by iron via the IRE-IRP system and is important for ferritin secretion by extracellular vesicles. Blood 2021;138:1490-503.

33. Toyokuni S. Role of iron in carcinogenesis: cancer as a ferrotoxic disease. Cancer Sci 2009;100:9-16.

34. Mobarra N, Shanaki M, Ehteram H, Nasiri H, Sahmani M, Saeidi $M$, et al. A review on iron chelators in treatment of iron overload syndromes. Int J Hematol Oncol Stem Cell Res 2016;10:239-47.

35. Zheng H, Jiang L, Tsuduki T, Conrad M, Toyokuni S. Embryonal erythropoiesis and aging exploit ferroptosis. Redox Biol 2021;48:102175.

36. Mori M, Ito F, Shi L, Wang $\mathrm{Y}$, Ishida $\mathrm{C}$, Hattori $\mathrm{Y}$, et al. Ovarian endometriosis-associated stromal cells reveal persistently high affinity for iron. Redox Biol 2015;6:578-86.

37. Kajiyama H, Suzuki S, Yoshihara M, Tamauchi S, Yoshikawa N, Niimi K, et al. Endometriosis and cancer. Free Radic Biol Med 2019;133:186-92.

38. Hayashi S, Nakamura T, Motooka Y, Ito F, Jiang L, Akatsuka S, et al. Novel ovarian endometriosis model causes infertility via ironmediated oxidative stress in mice. Redox Biol 2020;37:101726.

39. Torti SV, Manz DH, Paul BT, Blanchette-Farra N, Torti FM. Iron and cancer. Annu Rev Nutr 2018;38:97-125.

40. Toyokuni S. Novel aspects of oxidative stress-associated carcinogenesis. Antioxid Redox Signal 2006;8:1373-7.

41. Toyokuni S. Mysterious link between iron overload and CDKN2A/2B. J Clin Biochem Nutr 2011;48:46-9.

42. Akatsuka S, Yamashita Y, Ohara H, Liu YT, Izumiya M, Abe K, et al. Fenton reaction induced cancer in wild type rats recapitulates genomic alterations observed in human cancer. PLoS One 2012; 7:e43403.

43. Toyokuni S. The origin and future of oxidative stress pathology: from the recognition of carcinogenesis as an iron addiction with ferroptosis-resistance to non-thermal plasma therapy. Pathol Int 2016;66:245-59.

44. Toyokuni S, Ito F, Yamashita K, Okazaki Y, Akatsuka S. Iron and thiol redox signaling in cancer: an exquisite balance to escape ferroptosis. Free Radic Biol Med 2017;108:610-26.

45. IARC, WHO. Asbestos (chrysotile, amosite, crocidolite, tremolite, actinolite and anthophyllite). In: IARC Working Group on the 
Evaluation of Carcinogenic Risks to Humans, World Health Organization, eds. A Review of Human Carcinogens: Part C: Arsenic, Metals, Fibres, and Dusts. Lyon, International Agency for Research on Cancer, pp 219-309, 2012.

46. Oury TD, Sporn TA, Roggli VL. Pathology of asbestos-associated diseases. 3rd ed. Berlin/Heidelberg, Springer, 2014.

47. Toyokuni S. Mechanisms of asbestos-induced carcinogenesis. Nagoya J Med Sci 2009;71:1-10.

48. Toyokuni S. Iron addiction with ferroptosis-resistance in asbestos-induced mesothelial carcinogenesis: toward the era of mesothelioma prevention. Free Radic Biol Med 2019;133:20615.

49. Toyokuni S, Ito F, Motooka Y. Role of ferroptosis in nanofiberinduced carcinogenesis. Metallomics Res 2021;1:14-21.

50. Nagai $H$, Ishihara $T$, Lee WH, Ohara H, Okazaki Y, Okawa K, et al. Asbestos surface provides a niche for oxidative modification. Cancer Sci 2011;102:2118-25.

51. Nagai $H$, Toyokuni S. Differences and similarities between carbon nanotubes and asbestos fibers during mesothelial carcinogenesis: shedding light on fiber entry mechanism. Cancer Sci 2012;103:1378-90.

52. Xio S, Li D, Vijg J, Sugarbaker DJ, Corson JM, Fletcher JA. Codeletion of $\mathrm{p} 15$ and $\mathrm{p} 16$ in primary malignant mesothelioma.

\section{Oncogene 1995;11:511-5.}

53. Jiang L, Akatsuka S, Nagai H, Chew SH, Ohara H, Okazaki Y, et al. Iron overload signature in chrysotile-induced malignant mesothelioma. J Pathol 2012;228:366-77.

54. Stanton MF, Wrench C. Mechanisms of mesothelioma induction with asbestos and fibrous glass. J Natl Cancer Inst 1972;48:797821.

55. Ito F, Yanatori I, Maeda Y, Nimura K, Ito S, Hirayama T, et al. Asbestos conceives $\mathrm{Fe}(\mathrm{II})$-dependent mutagenic stromal milieu through ceaseless macrophage ferroptosis and $\beta$-catenin induction in mesothelium. Redox Biol 2020;36:101616.

56. Ito F, Kato K, Yanatori I, Murohara T, Toyokuni S. Ferroptosisdependent extracellular vesicles from macrophage contribute to asbestos-induced mesothelial carcinogenesis through loading ferritin. Redox Biol 2021;47:102174.

57. Rezania S, Park J, Md Din MF, Mat Taib S, Talaiekhozani A, Kumar Yadav K, et al. Microplastics pollution in different aquatic environments and biota: a review of recent studies. Mar Pollut Bull 2018;133:191-208.

58. Sana SS, Dogiparthi LK, Gangadhar L, Chakravorty A, Abhishek $\mathrm{N}$. Effects of microplastics and nanoplastics on marine environment and human health. Environ Sci Pollut Res Int 2020;27:44743-56. 